[Agr. Biol. Chem., Vol. 36, No. 9, p. 1639 1642, 1972]

\title{
Maillard Reaction Products Formed from L-Rhamnose and Ethylamine
}

\author{
By Hiromichi Kato, Hitoshi Shigematsu," Tadao Kurata \\ and Masao Fujimaki \\ Department of Agricultural Chemistry, The University \\ of Tokyo, Bunkyo-ku, Tokyo \\ *Central Research Institute, Japan Monopoly. \\ Corporation, Shinagawa-ku, Tokyo \\ Received February 7, 1972
}

In the previous papers, ${ }^{1-9)}$ the authors described the formation of $\mathrm{N}$-substituted pyrrole-2-aldehydes in the Maillard reaction between sugars and alkylamines or amino acids. In the present paper, the volatile products formed by the reaction between Lrhamnose and ethylamine are investigated.

\section{Isolation and characterization of the reaction products}

The mixture of L-rhamnose and ethylamine acetate was heated in an aqueous solution (initial $\mathrm{pH}$ 6.0) for $1 \mathrm{hr}$ at $100^{\circ} \mathrm{C}$ and the resulting Maillard reaction products were extracted with ethyl acetate. The extract was fractionated by preparative gas chromatography. The gas chromatogram was as shown in Fig. 1. The four main peaks, peak $1,3,4$ and 5 , were trapped separately.

The main component in peak 1 was identified as acetic acid by the agreement of its aroma and retention time with those of authentic sample. Peak 2, a small peak between peak 1 and 3 , was not isolated but tentatively identified as 5-methyl-2-furaldehyde (I) by comparison of the retention time with that of the authentic sample prepared as described by Taylor. ${ }^{4 ;}$ Peak 5 , the most abundant peak, was very fragrant and was proved to be 2,5dimethyl-4-hydroxy-3(2H)-furanone (II) by

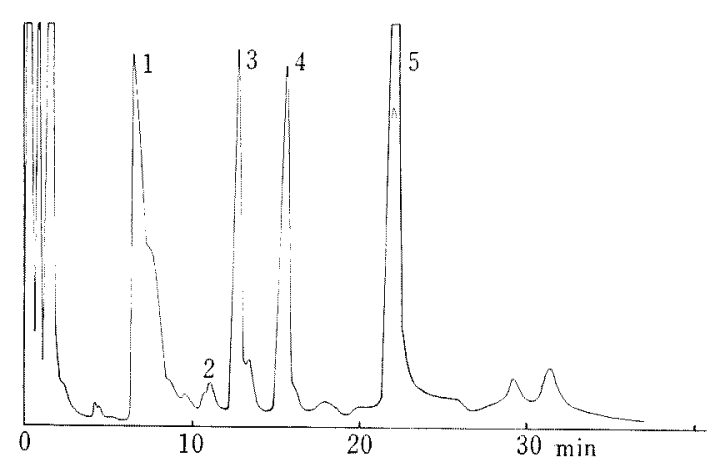

Fic. 1. Gas Chromatogram of the Extract from the Maillard Reaction Products between L-Rhamnose and Ethylamine.

comparison of IR and MS spectra with those described by Rodin et al..$^{5)} \quad$ The yield of this compound was $c a .5 \%$ of the rhamnose used, which was calculated from the peak area.

The compound in peak 4 showed a carbonyl group at $1656 \mathrm{~cm}^{-1}$ in its IR spectrum and the parent peak at $m / e 137$ in its MS spectrum. NMR spectrum of this peak was as follows: NMR $\tau_{\mathrm{Nu}_{4} \mathrm{Si}_{3}}^{\mathrm{CDCl}_{3}}, 8.71,5.68(3 \mathrm{H}$, triplet; $2 \mathrm{H}$, quartet; $\left.J=7 \mathrm{HZ},>\mathrm{N}-\mathrm{CH}_{2} \mathrm{CH}_{3}\right), 7.72(3 \mathrm{H}$, singlet, $\left.-\mathrm{CH}_{3}\right), 4.01,3.19(1 \mathrm{H}$, doublet; $1 \mathrm{H}$, doublet; $J=3.5 \mathrm{HZ}$, two protons attached to pyrrole ring), $0.62(1 \mathrm{H}$, singlet, $-\mathrm{CHO})$. These spectroscopic data indicate that this compound is the pyrrolealdehyde expected to be formed 

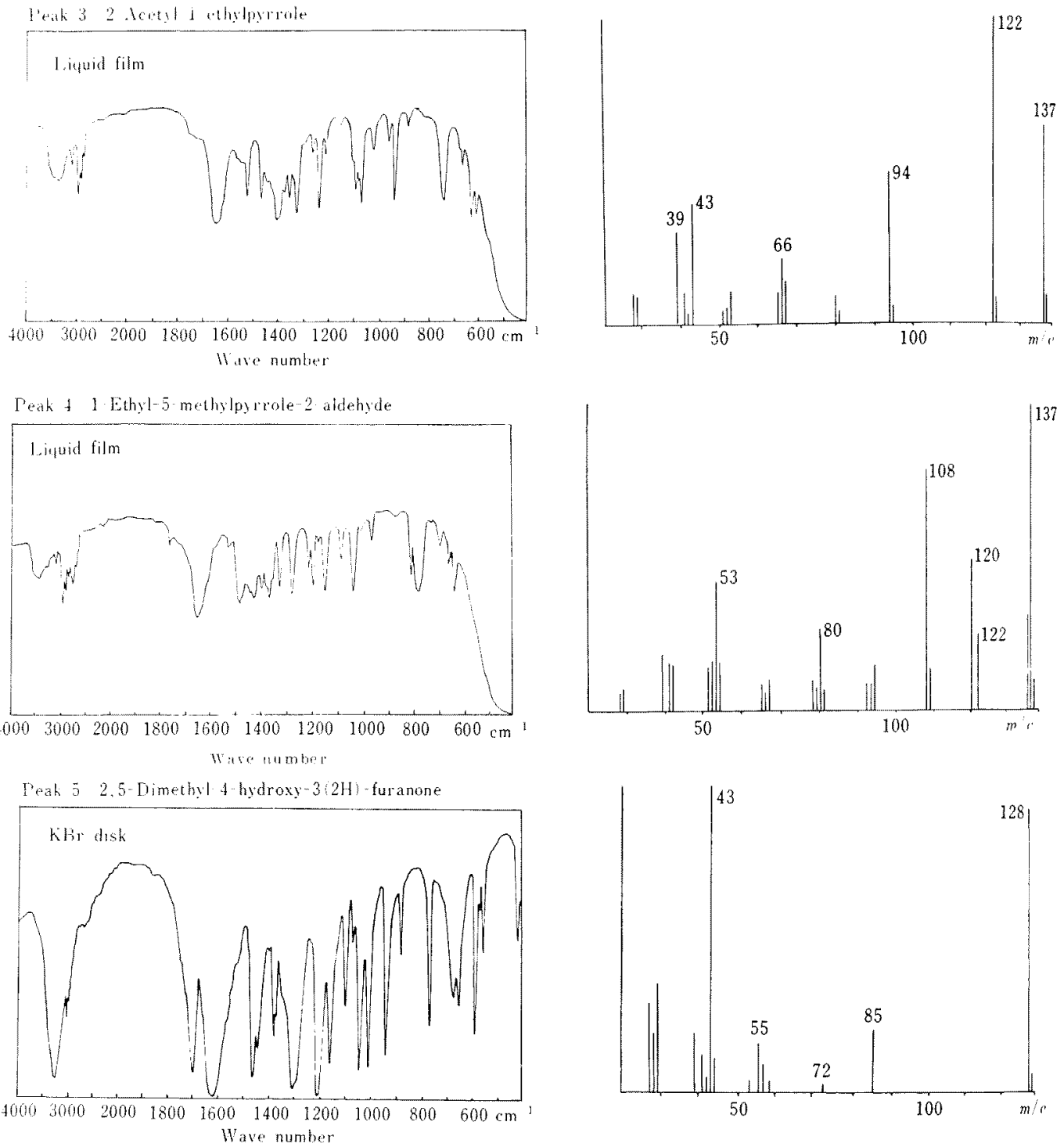

FIG. 2. IR and MS Spectra of Peak 3, 4 and 5.

from rhamnose-ethylamine system, i.e. 1-ethyl5-methylpyrrole-2-aldehyde (III).

On the other hand, the compound in peak 3 also showed a carbonyl group at $1650 \mathrm{~cm}^{-1}$ in its IR spectrum and the MS spectrum of this peak revealed $m / e 137\left(\mathrm{M}^{+}\right)$and the presence of an acetyl group; NMR spectrum was as follows: NMR $\tau_{\mathrm{Mc}_{4} \mathrm{~S}_{\mathrm{i}}}^{\mathrm{CDCl}_{3}}, 8.64,5.39(3 \mathrm{H}$, triplet; $2 \mathrm{H}$, quartet; $J=7 \mathrm{HZ}, \mathrm{N}-\mathrm{CH}_{2} \mathrm{CH}_{3}$ ), $7.57\left(3 \mathrm{H}\right.$, singlet, $\left.-\mathrm{COCH}_{3}\right), 3.90,3.2 \sim 3.0$ (1H, quartet; $2 \mathrm{H}$, two quartets; three protons attached to pyrrole ring). From these spectroscopic results, it is concluded that this compound is 2-acetyl-1-ethylpyrrole (IV). Both IR and MS spectra of peak 3, 4 and 5 are shown in Fig. 2. 


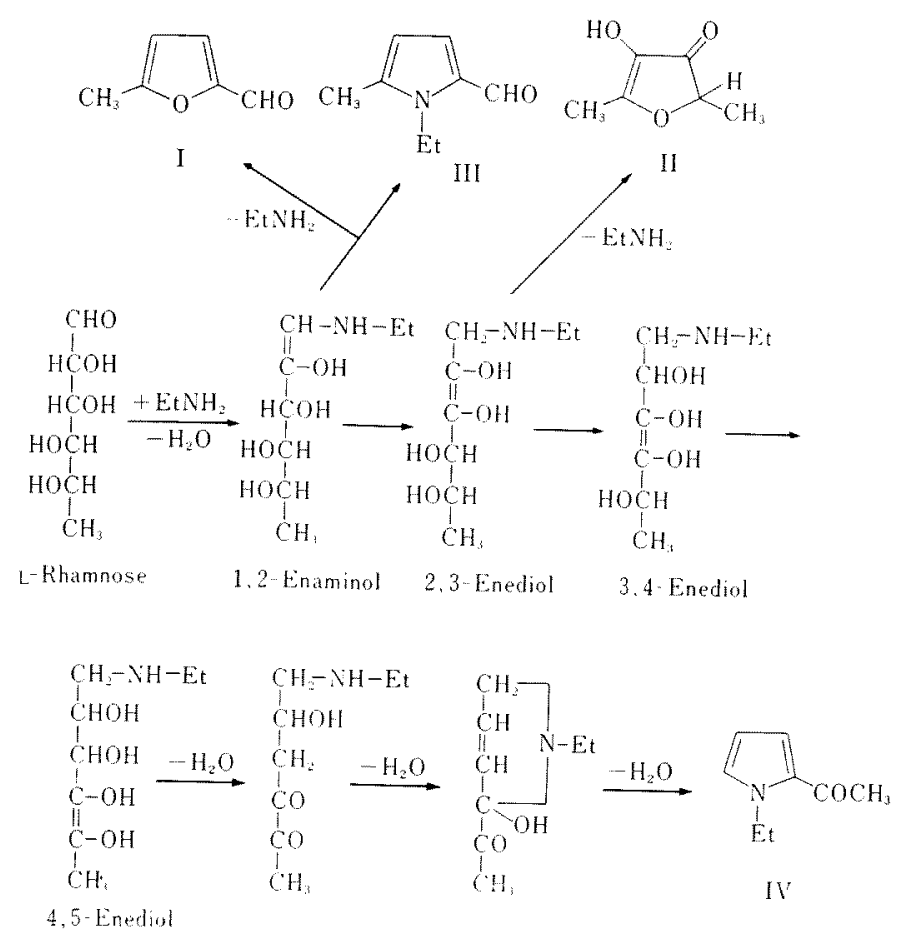

FIG. 3. The Proposed Formation Pathway of 2-Acetyl-1-ethylpyrrole (IV) and the Other Reaction Products $(I \sim$ III).

Formation pathways of the isolated compounds

Pyrrolealdehyde (III) is formed via 1,2enaminol of 1-amino-1-deoxyketose and 3deoxyosulose as described in the previous paper. ${ }^{1\rangle}$ Furaldehyde (1) also is formed via the same pathway. On the other hand, furanone (II) is formed via 2,3-enediol of 1-amino1-deoxyketose and methyl $\alpha$-dicarbonyl intermediate as proposed by Mills et al ${ }^{6 j}$ Hodge et al. ${ }^{77}$ prepared the furanone (II) from Lrhamnose and piperidine. The formation of II from L-rhamnose and ethylamine suggests that, 2,3-enolization of 1-amino-1-deoxyketose occurs in the reaction system between aldose and primary amine or amino acid as well as in the secondary amine reaction system, and further, 2,3-enolization is involved in the formation of melanoidin as well as 1,2-enolization is.
As described above, acetylpyrrole (IV) was formed; this type of nitrogen compound was isolated for the first time in the Maillard reaction system. If $\mathrm{C}-\mathrm{C}$ scission of rhamnose occurred under the present reaction condition, many pathways for the formation of IV would be possible. However,as one of the possible pathways without the $\mathrm{C}-\mathrm{C}$ scission, the authors propose a formation mechanism shown in Fig. 3 , in which the migration of enolization to 4,5 position is involved.

\section{EXPERIMENTAL}

Maillard reaction of L-rhamnose with ethylamine. L-Rhamnose $(5 \mathrm{~g})$ was dissolved in water $(10 \mathrm{ml})$ and acetic acid $(2.5 \mathrm{~g})$ was added. To the solution was added ethylamine ( $2 \mathrm{~g}, 70 \%$ aqueous solution) and the resulting mixture $(\mathrm{pH} 6.0$ ) was heated for $1 \mathrm{hr}$ in boiling water to obtain Maillard reaction products. 
The brownish black products were extracted with ethyl acetate four times. The extracts were combined, washed twice with sodium chloride-saturated aqueous solution, dried with anhydrous sodium sulfate and filtered. The filtrate was evaporated in vacuo and the resulting concentrate $(0.35 \mathrm{~g})$ was subjected to preparative gas chromatography.

Gas chromatography. The instrument employed was a Hitachi K-53 Gas Chromatograph equipped with a thermal conductivity detector. A stainless steel column, $1 \mathrm{~m} \times 3 \mathrm{~mm}$ (i.d.) packed with $20 \%$ Carbowax $20 \mathrm{M}$ on Chromosorb $\mathrm{W}(60 \sim 80$ mesh), was used for preparative gas chromatography. The operating conditions were as follows: carrier gas, helium with a flow rate of $40 \mathrm{ml} / \mathrm{min}$ at $80^{\circ} \mathrm{C}$; temperature programming, from $80^{\circ} \mathrm{C}$ to $250^{\circ} \mathrm{C}$ at $5^{\circ} \mathrm{C} / \mathrm{min}$; injection port temperature, $230^{\circ} \mathrm{C}$; detector temperature, $250^{\circ} \mathrm{C}$. Each peak was trapped in capillary glass tube cooled with dry ice-methanol.

Spectroscopic methods. IR spectra were recorded with a JASCO IR-G Spectrophotometer in liquid film or $\mathrm{KBr}$ disk. MS spectra were measured with a Hitachi RMU-7 Mass Spectrometer under the ioniz- ing voltage of $70 \mathrm{eV}$. NMR spectra were taken with a JNM 4H-100 Spectrometer in deuteriochloroform with tetramethylsilane as an internal standard.

Acknolwedgement. The authors are indebted to Mr. Yoichi Kaburaki, Japan Monopoly Corporation for his support.

\section{REFERENCES}

1) H. Kato, Agr. Biol. Chem., 30, 822 (1966); idem, ibid., 31, 1086 (1967).

2) H. Kato and M. Fujimaki, J. Food Sci, 33, 445 (1968).

3) H. Kato and M. Fujimaki, Agr. Biol. Chem., 34, $1071(1970)$.

4) D. A. H. Taylor, J. Chem. Soc., 1959, 2767.

5) J. O. Rodin, C. M. Himel, R. M. Silverstein, R. W. Leeper and W. A. Gortner, J. Food Sci., 30, 280 (1965).

6) F. D. Mills, B. G. Baker and J. E. Hodge, J. Agr. Food Chem., 17, 723 (1969).

7) J. E. Hodge, B. E. Fisher and E. C. Nelson, Am. Assoc. Brewing Chemists Proc,, 1963, 84. 